\title{
Changes in Expression of miRNA-320a and miRNA-497-5p in Early Stage of Breast Cancer
}

\author{
Zahra Nashtahosseini ${ }^{1}$, Farzin Sadeghi ${ }^{2}$ and Mahmoud R. Aghamaali ${ }^{3, *}$ \\ ${ }^{1}$ Department of Biology, University Campus 2, University of Guilan, Rasht, Iran \\ 2 Cellular and Molecular Biology Research Center, Health Research Institute, Babol University of Medical Sciences, Babol, Iran \\ ${ }^{3}$ Department of Biology, Faculty of Sciences, University of Guilan, Rasht, Iran
}

* Corresponding author: Mahmoud R. Aghamaali, Department of Biology, Faculty of Sciences, University of Guilan, Rasht, Iran. Tel: +981333690274; Fax: +981333690275; Email: aghamaali@guilan.ac.ir

Received 2021 March 01; Revised 2021 April 15; Accepted 2021 May 18.

\begin{abstract}
Background: Micro-ribonucleic acids (miRNAs) are noncoding small RNA species considered a varying class with a single-stranded structure whose expression is often dysregulated in cancer. The expression of miRNAs has been used as a promising new biomarker for the detection of breast cancer (BC).

Objectives: The purpose of the present case-control study was to investigate the expression levels of miRNA-320a and miRNA-497-5p and their potential role in $\mathrm{BC}$ patients in comparison to those of the healthy controls in the Cellular and Molecular Biology Research Center, Health Research Institute, Babol University of Medical Sciences, Babol, Iran, in 2019.

Methods: The concentrations of miR-320a and miR-497-5p were analyzed in 80 serum samples of 40 patients with a confirmed diagnosis of early-stage BC in comparison to those of 40 age-matched healthy volunteers. Real-time quantitative polymerase chain reaction was carried out for the detection of the expression level of these miRNAs.

Results: The results of the current study showed that the serum levels of miR-320a and miR-497-5p were down-regulated in the BC patients, compared to those reported for the healthy controls $(\mathrm{P}=0.651$ and $\mathrm{P}=0.044$, respectively). However, the levels of mi $\mathrm{R}-320 \mathrm{a}$ in the early-stage BC samples were not statistically different from those of the healthy volunteers. There was a reduction in the serum miRNA-320a of the premenopausal subjects under 48 years of age. Serum miRNA-497-5p also decreased among the cases under 48 years of age.

Conclusion: The identification and effectiveness of these miRNAs were demonstrated in the early-stage BC screening. It seems that miRNAs have the potential to be used as biomarkers for the screening and diagnosis of BC.
\end{abstract}

Keywords: Breast cancer, MicroRNA, MicroRNA-320a, MicroRNA-497-5p, Real-time PCR, Serum

\section{Background}

Breast cancer $(\mathrm{BC})$ is considered the most prevalent cancer causing mortalities among females. Genetic alterations, diet, hormones, environmental factors, toxins, and stress are the predominant reasons for BC. This disease consists of several subtypes differing in their molecular characteristics (1); however, the early diagnosis of $\mathrm{BC}$ is effective in the improvement of these patients' health conditions similar to other cancers (2).

Micro-ribonucleic acids (miRNAs) are regarded as conserved, small nucleotides, and non-coding RNAs, with an essential function for the regulation of gene expression (3). The miRNAs in a nuclear protein complex called the RNA-induced silencing complex interact with the three untranslated regions of target messenger RNAs (mRNAs) at specific sites to induce the degradation and translational repression of mRNA $(4,5)$.

In a recent study, the dysregulation of microRNAs was included in the initiation and progression of different human cancers, such as BC $(6,7)$. Therefore, the examination of the changes in miRNAs in cancers provides the possibility for the diagnosis/prognosis of specific cancers and differentiation of specific types of cancer (8). Abundant evidence has demonstrated that miRNAs can act as oncogenes or tumor suppressor genes in various cancers $(9,10)$, and this is dependent on the up- or down-regulation of miRNAs in human cancers (11).

The miRNAs are in the body fluid similar to the serum, and the relative ease of detection in addition to their remarkable stability make circulating miRNAs perfect tools for quick and non-invasive diagnosis (12). The recognition of the molecular mechanisms involved in the initiation and progression of $\mathrm{BC}$ is useful in the development of strategies for the early diagnosis of $\mathrm{BC}$ and treatment of this deadly disease.

The miR-320a behaves as a tumor suppressor and has been down-regulated in $\mathrm{BC}$ tissue. In addition, the down-regulation of miR-320a increases the overexpression of its target gene Rab11a via the mechanism involving the inactivation of protein kinase B signaling. The expression of Rab11a was up-regulated in tumor samples (13). The over-expression of miR-497-5p leads to suppressing Bcl-w expression at mRNA and protein levels, functioning as a tumor suppressor through targeting pro-apoptotic genes and Bcl-w in BC, inhibiting the growth, and simplifying the apoptosis and G0/G1 phase arrest (14). 
The results of a study suggested that miR-320a presents a potential biomarker for the prognosis of invasive $\mathrm{BC}$ and when dysregulated may be involved in invasive BC progression (15). Another study reported a significant difference in the levels of miR320 a between the patients with low tumor-stage of prostate cancer in comparison to those reported for subjects with high tumor-stage (16). Luo et al. demonstrated the down-regulation of miR-320 expression in $\mathrm{BC}$ tissues in comparison to that reported for adjacent normal tissues and the possibility of serving as a prognostic biomarker (17). Another study carried out by Bai et al. indicated the functioning of miR-320 as a tumor suppressor in tumorigenesis and progression of BC (18). In another study conducted by Zhang et al., it was reported that miR-320 can be a good prognostic biomarker for cervical cancer (19).

The miR-497-5p was firstly reported in human BC, and the down-regulation of miR-497-5p has been shown in different carcinomas, such as BC (20). Another study carried out by Hou et al. demonstrated that the relative expression level of miR-497 in $\mathrm{BC}$ tissues was significantly lower than that in the corresponding noncancerous breast tissues (21). It seems that the expression of miR-497-5p was significantly down-regulated in BC tissue samples and cell lines $(14,22)$.

The present study assayed the changes in the serum levels of miR-320a and miR-497-5p among individuals with early-stage $\mathrm{BC}$ in comparison to those of the healthy controls. However, there have been a limited number of studies reporting the clinicopathological value and prognostic significance of miR-320a and miR-497-5p expression in BC. Furthermore, the current study firstly demonstrated that the expression levels of miR-320a and miR-497$5 p$ were lower in the early-stage BC specimens, compared to those reported for the serum of normal samples. In addition, there was an interest in the investigation of the diagnostic precision of these miRNAs in order to distinguish between the patients and healthy individuals.

\section{Objectives}

The current study aimed to define whether the serum levels of these miRNAs in the early-stage of BC vary similar to those in the advanced stage of the disease and these microRNAs can be useful as biomarkers in the early diagnosis of BC. Furthermore, there was an interest in the examination of the diagnostic accuracy of these miRNAs in order to distinguish between the patients and healthy individuals.

\section{Methods}

In this case-control study, the miRNAs levels of
miR-320a and miR-497-5p were analyzed in the serum of 40 females with a confirmed diagnosis of early-stage $\mathrm{BC}$ and 40 healthy controls within the age range of 36-77 years. The current study was conducted on all the patients referred to the affiliated hospitals of Babol University of Medical Sciences, Babol, Iran, within May 2016 to February 2017. There was a previous report on the status of miRNAs in the aforementioned samples $(23,24)$.

In this study, $5 \mathrm{~mL}$ of peripheral blood samples were gathered in regular diagnostic assessments in a serum separator tube prior to any treatment (e.g., surgery, radiation, or systemic therapy). The whole blood was permitted to stay for about $1 \mathrm{~h}$ at room temperature previous to centrifugation at 3,500 rpm for $10 \mathrm{~min}$. The consequent serum was aliquoted into Eppendorf tubes and stored at $-80^{\circ} \mathrm{C}$.

The inclusion criteria of the present study were the female gender, diagnosis and confirmation by pathology, and BC at an early stage (i.e., 0, I, II, and III). Any patients received preoperative adjuvant chemotherapy or radiotherapy on the basis of the clinical staging method. In addition, no other cancer or diseases was reported among the patients with a possibility of affecting miRNA profiling. Furthermore, individuals with a history or known symptoms of cardiovascular disease, chronic kidney disease, Crohn's disease, diabetes, rheumatoid arthritis, osteoarthritis, and other cancers were excluded from the study. Moreover, the patients with blood donation within the previous 3 years were ruled out from the study. The volunteers in this study were healthy subjects with normal mammography and ultrasound findings with no history of malignant diseases.

It was also possible to have access to the clinical and pathological data of all the samples of this study, including age at diagnosis, menopausal status, tumor pathology, stage, grade, estrogen receptor (ER) status, progesterone receptor (PR) status, and human epidermal growth factor receptor 2 (HER2) expression. The determination of ER, PR, and HER2 status were on the basis of immune histochemical assays carried out in order to provide routine clinical care. A breast pathologist evaluated the histology of the tumor and tissue. The present study was approved by the Ethics Committee of Babol University of Medical Sciences. In addition, all the study procedures were explained to the participants, and then written informed consent was obtained from all the patients.

\subsection{RNA extraction}

The RNA extraction was performed at Babol University of Medical Sciences in 2018. The small RNA molecules of the serum were extracted using TRIzol Reagent (Invitrogen, Carlsbad, CA, USA) based on the manufacturer's instructions. The RNA was eluted by RNase-free water in a final volume of 
$30 \mu \mathrm{L}$ and stored at $-80^{\circ} \mathrm{C}$ until use. The quality of RNA was recognized using a NanoDrop spectrophotometer (Thermo Fisher Scientific, Wilmington, USA).

\section{2. $c D N A$ synthesis}

This step was carried out by a peqSTAR $2 \mathrm{X}$ Gradient Thermocycler (Peqlab Biotechnologie GMBH, Erlangen, Germany). The RNA molecules were reverse-transcribed using a particular stem-loop primer for miR-320a, miR-497-5p, and mir-16 analysis (Metabion International AG, Germany), as shown in Table 1 . The $15-\mu$ l reverse transcription reaction contained $160 \mathrm{ng}$ of total RNA, $5 \mathrm{pmol} / \mu \mathrm{l}$ of each stem-loop primer, $7.5 \mu \mathrm{l}$ of $2 \mathrm{X}$ reverse transcriptase reaction buffer (GeNet Bio, SuPrimeScript RTase, Korea), $1.5 \mu \mathrm{l}$ each of deoxynucleotide triphosphates (GeNet Bio, SuPrimeScript RTase), $1 \mu \mathrm{l}$ (100 U) of Moloney murine leukemia virus reverse transcriptase (GeNet Bio, SuPrimeScript RTase), and diethyl-pyrocarbonatetreated water (GeNet Bio, SuPrimeScript RTase) to get to the total reaction volume. Accordingly, the reaction was carried out at the temperatures of $16^{\circ} \mathrm{C}$ for 30 $\min , 50^{\circ} \mathrm{C}$ for $30 \mathrm{~min}$, and $70^{\circ} \mathrm{C}$ for $10 \mathrm{~min}$, after which the sample was maintained at $4^{\circ} \mathrm{C}$.

\subsection{Real-time polymerase chain reaction}

Real-time polymerase chain reaction (PCR) was performed by means of a miScript SYBR Green PCR kit (Qiagen, Germany) and assayed with the primer sets of miR-320a and miR-497-5p as the target genes and miR-16 as a housekeeping gene shown in Table 1 . In addition, real-time PCR was carried out and detected by QIAGEN's Real-time PCR system cycler (the RotorGene Q; Qiagen, Germany). Accordingly, $1 \mu \mathrm{L}$ of complementary deoxyribonucleic acid (cDNA) was mixed as a template in $20 \mu \mathrm{L}$ of total volume reaction, $10 \mu \mathrm{L}$ of Master Mix Amplicons (Real Q Plus 2x Master Mix Green without ROX ; Ampliqon, Odense, Denmark), and $5 \mathrm{pmol} / \mu \mathrm{l}$ of each primer. The realtime PCR reaction was performed in the duplication of denaturation at $95^{\circ} \mathrm{C}$ for $15 \mathrm{~min}$ by 40 cycles of $95^{\circ} \mathrm{C}$ for $12 \mathrm{sec}$ and $60^{\circ} \mathrm{C}$ for $25 \mathrm{sec}$.

Two target miRNAs (i.e., miR-320a and miR-497$5 p$ ) and housekeeping miRNA (i.e., miR-16) in duplicates were used to validate the differentially expressed miRNAs that were identified in the discovery set. In addition, the standard deviation was calculated. Master mix without cDNA as a negative control was utilized for the investigation of the contamination. Subsequently, melting curve analysis and gel electrophoresis were employed to confirm the amplification specificity of the expected PCR product, and there were no nonspecific products Table 1 tabulates the miRNAs specific primer sequences. The miR-16 as an endogenous control was utilized by relative expression software tool (REST; version 2008) for normalized miR-320a and miR-497-5p. For the determination of the dependent quantification of a target gene in comparison to a reference gene, the relative expression ratio (R) of a target gene was used, which is estimated based on the Ct (cycle threshold) and efficiency (E) deviation of unknown samples in comparison to those of the controls. The miR-16 was used for the normalization of the relative expression of miR-320a and miR-497-5p, which were calculated using the Pfaffl method (25).

\subsection{Statistical analysis}

The Shapiro-Wilk test was utilized in order to check the normality of the distribution of miR-320a and miR-497-5p expression levels. Then, the data were analyzed using the Mann-Whitney $U$ test, Kruskal-Wallis test, and Chi-square test. For the comparison of the age mean values between the two groups of the study, the independent samples t-test was employed. Furthermore, in the statistical analysis, the stages were classified into early (i.e., in situ, I, and II) and advanced (i.e., III) according to the tumor, node, and metastasis (TNM) staging system. All computational statistics and data analysis were conducted by SPSS software (version 23.0). Moreover, a p-value of less tha n 0.05 was considered statistically significant.

\begin{tabular}{|c|c|c|c|}
\hline & Oligo ID & Primer name & Sequence $\left(5^{\prime}-3^{\prime}\right)$ \\
\hline \multirow{2}{*}{ MI0000542 } & $\begin{array}{l}\text { 171201X36B05 } \\
41 / 118\end{array}$ & miR-320a-RT & $\begin{array}{l}\text { GTCGTATCCAGTGCAGGGTCCGAGGTATTCGCACTGGAT } \\
\text { ACGACTCGCCC }\end{array}$ \\
\hline & $\begin{array}{l}\text { 171201B004A12 } \\
42 / 118\end{array}$ & miR-320a-F & AAGCTGGGTTGAGAGGGC \\
\hline \multirow{2}{*}{ MIMAT0002820 } & $\begin{array}{l}\text { 171201X036B04 } \\
39 / 118\end{array}$ & miR-497-5p-RT & $\begin{array}{l}\text { GTCGTATCCAGTGCAGGGTCCGAGGTATTCGCACTGGAT } \\
\text { ACGACACAAAC }\end{array}$ \\
\hline & $\begin{array}{l}\text { 171201B004B12 } \\
40 / 118\end{array}$ & $\mathrm{miR}-497-5 \mathrm{p}-\mathrm{F}$ & CAGCAGCACACTGTGGTTTG \\
\hline \multirow{3}{*}{ MIMAT0000069 } & $171201 X 036 \mathrm{~B} 0645 / 118$ & miR-16-5p-RT & GTCGTATCCAGTGCAGGGTCCGAGGTATTCGC \\
\hline & 171201Х036B06 46/118 & miR-16-5p-Forward & GGTAGCAGCACGTAAATATTGGCG \\
\hline & 171201B004H11 43/118 & Universal-Reverse & GTGCAGGGTCCGAGGT \\
\hline
\end{tabular}




\section{Results}

\subsection{Patient characteristics}

The current study was carried out on a total of 80 female participants, including 40 subjects with earlystage BC and 40 healthy individuals. Table 2 tabulates the clinical features of the BC participants and cancer pathology. The age mean value of the healthy subjects was reported as $48.18 \pm 6.18$ years, which was significantly lower than that $(51.0 \pm 10.20$ years $)$ reported for the early-stage $\mathrm{BC}$ patients $(\mathrm{P}=0.005)$. The stages of $B C$ were reported as $0(n=1 ; 2.5 \%)$, I $(\mathrm{n}=5 ; 12.5 \%)$, II $(\mathrm{n}=19 ; 47.5 \%)$, and III $(\mathrm{n}=15 ; 37.5 \%)$. In addition, 15 (37.5\%) and $25(62.5 \%)$ BC cases were invasive and in situ, respectively.

\subsection{MiRNA as biomarkers for breast cancer detection}

The present study assessed the serum levels of hsa-miR-320a and miR-497-5p because these miRNAs were significantly under-expressed in BC tissues, compared to those of the healthy ones. The MiR-16 was used as one of the methods of normalization. In the current study, it was aimed to determine whether the levels of miR-320a and miR497-5p in the early stages of the BC group differ from those of the healthy group and this microRNA can be introduced as a probable diagnostic biomarker.

During the analysis, the concentration of miR$320 \mathrm{a}$ and miR-497-5p were analyzed in the serum samples of 40 patients with early-stage BC. In comparison to those reported for the healthy volunteers, there was a reduction in the serum levels of miR-320a and miR-497-5p in the patient samples. However, these results indicated that the levels of miR-320a in the patient samples were not statistically different from those reported for the healthy volunteers $(\mathrm{P}=0.651)$. In addition, as illustrated in Figure 1, there was a significant difference in miR-497-5p between the patients and healthy volunteers $(\mathrm{P}=0.044)$.

Therefore, the levels of these miRNAs were analyzed in the patients with early-stage BC and healthy volunteers for the evaluation of the effects of miR-320a and miR-497-5p expression levels and changes in the patient group. Nevertheless, after the identification of the impact of the menopausal condition on the serum level in premenopausal women, serum miR-320a and miR-497-5p levels in the BC subjects were significantly lower in comparison to those reported for the controls; however, the postmenopausal patients were reported with a significantly lower level of miR-497-5p in comparison to the healthy individuals.

According to the results of the present study, miR-320a and miR-497-5p were significantly downregulated in the $\mathrm{BC}$ group in comparison to those of the healthy individuals ( 2.56 and 1.32 fold change decrease, respectively). In both various age groups (i.e., $<48$ and $\geq 48$ years), the expression levels of

\begin{tabular}{|c|c|c|c|}
\hline & Breast cancer $(n=40)$ & Healthy $(n=40)$ & P-value \\
\hline Age (year) mean \pm standard deviation & $51.0 \pm 10.20$ & $48.18 \pm 6.18$ & 0.005 \\
\hline \multicolumn{4}{|l|}{ Cancer stage (n) } \\
\hline 0 & 1 & & \\
\hline $\mathrm{I}$ & 5 & & \\
\hline II & 19 & & \\
\hline III & 15 & & \\
\hline \multicolumn{4}{|l|}{ Cancer subtype (\%) } \\
\hline Invasive & 37.5 & & \\
\hline In situ & 62.5 & & \\
\hline
\end{tabular}

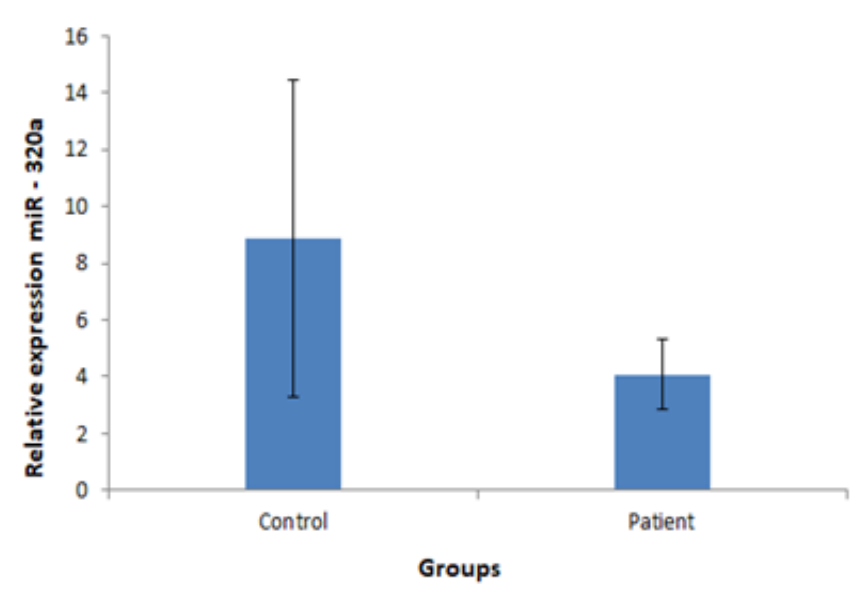

Figure 1. Relative expression levels of circulating micro-ribonucleic acids in control and patient groups; evaluation of serum level of miR-320a using real-time polymerase chain reaction (RT-PCR); determination of a statistically significant difference using the Mann-Whitney U test 


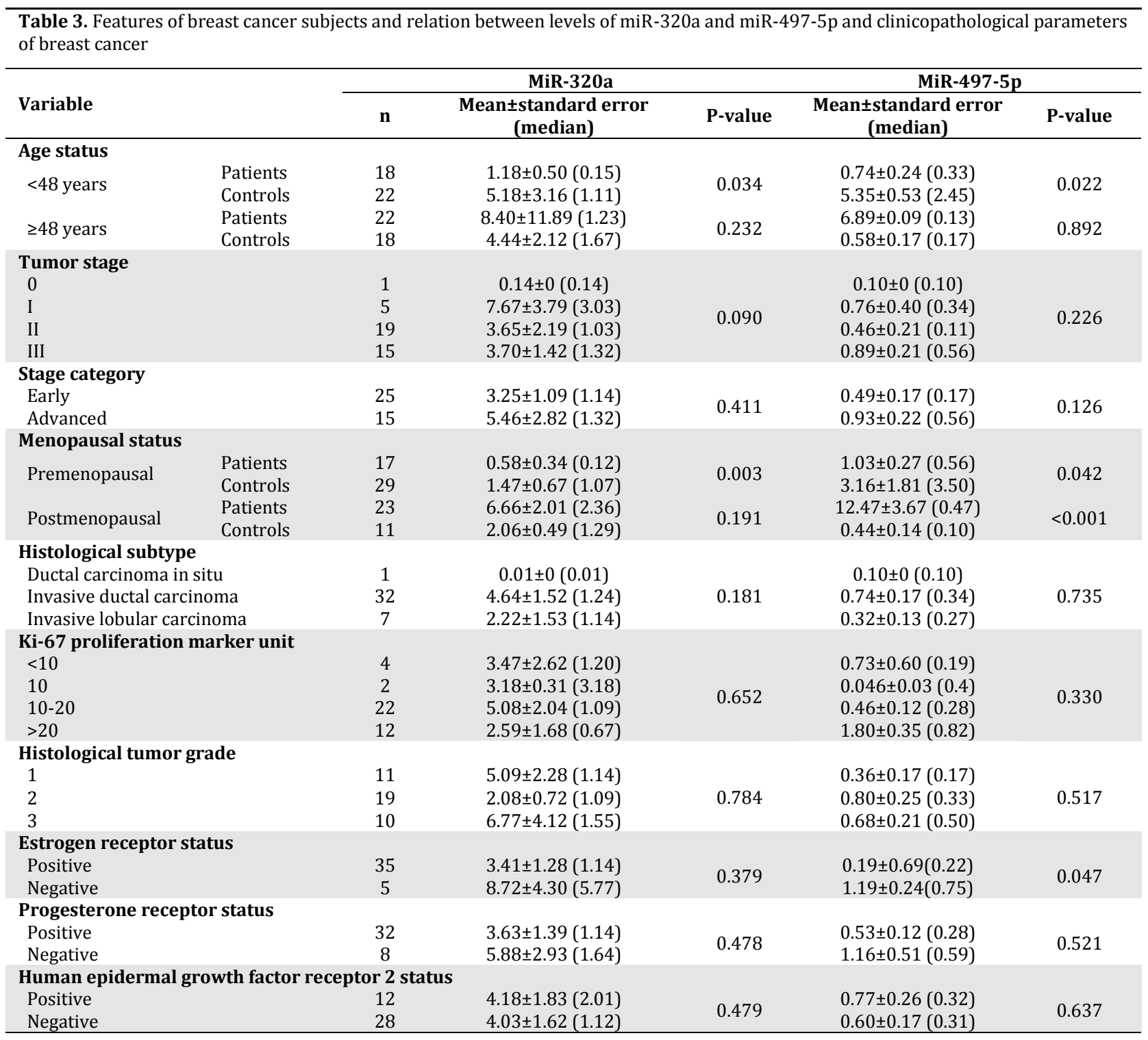

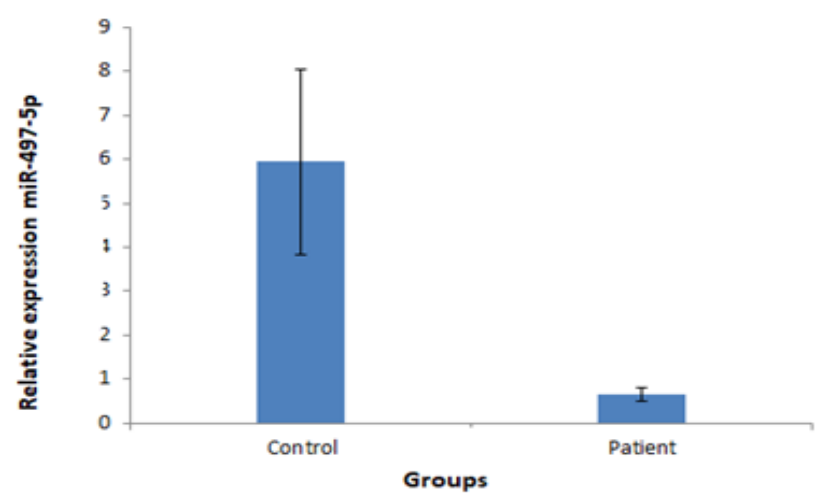

Figure 2. Relative expression levels of circulating micro-ribonucleic acids in control and patient groups; evaluation of a serum level of miR-497-5p using real-time polymerase chain reaction (RT-PCR);; determination of a statistically significant difference using the Mann-Whitney U test

these miRNAs in the age category of $<48$ years, were significantly lower in the patients with $\mathrm{BC}$ subjects in comparison to those reported for the healthy individuals. As shown in Table 3, no 
significant difference was observed in miR-320a and miR-497-5p between HER2 positive and negative, ER positive and negative, and PR positive and negative among the patients with $\mathrm{BC}$.

\subsection{Clinicopathological features}

Table 3 tabulates the clinicopathological features of 40 subjects at the time of $\mathrm{BC}$ diagnosis. There were no significant differences between the serum levels of miR-320a and miR-497-5p and clinicopathological features and early and advanced stages of BC.

\section{Discussion}

The ability for the detection of cancer in the early stages is a major factor in the reduction of mortalities and improvement of patient survival. The determination of biomarkers for the diagnosis and prognosis of cancer is a certain advantage. Serum miRNAs considered a group of new promising noninvasive biomarkers are easy to be detected and resistant in hard conditions $(26,27)$. The present study aimed to determine whether the levels of miR$320 a$ and miR-497-5p in the early stages of the BC group differ from those of the healthy group and these microRNAs could be introduced as probable diagnostic biomarkers. To the best of our knowledge, the current study has been the first attempt regarding the use of serum miR-320a and miR-497$5 p$ levels for the differentiation of early-stage BC patients from healthy controls.

Previous studies investigated the changes in the expression levels of these microRNAs in cell culture. In a study carried out by Yang et al., it was shown that miR-320a was down-regulated in invasive BC, and miR-320a staining was observed to significantly correlate with clinicopathological features $(\mathrm{P}=0.047)$ (15). Luo et al. demonstrated that the down-regulation of miR-320a can lead to the enhanced expression of Aquaporin-1 in BC, and miR-320a can play the main role as a biomarker for prognosis and therapeutic targets in BC (17). In another study, Bai et al. reported that miR-320 decreased in $\mathrm{BC}$ cells and prevents cell proliferation and invasion in BC cells (18).

In a study carried out by Lieb et al., a significant difference was observed in the level of miR-320a among the subjects with low tumor-stage prostate cancer in comparison to the patients with high tumor-stage $(\mathrm{P}=0.034)$ and in cases with low serum prostate-specific antigen (PSA) in comparison to the subjects with high serum PSA ( $\leq 4$ vs. $>4$ ng; $P=0.003$ ) (16). Zhang et al. reported that miR-320 expression was significantly down-regulated in cervical cancer. Furthermore, there was a negative correlation between miR-320 expression and Mcl-1 expression. In addition, Zhang et al. concluded that miR-320 can be an important biomarker for the diagnosis and treatment of cervical cancer (19). .In another study, YAN et al. reported that compared to normal tumor tissues, miR-320 was down-regulated more than twofold change in primary stages of human BC(28). In a study carried out by Chang et al., it was reported that miR-497-5p and miR-195-5p in the miR-497-195 cluster at $17 \mathrm{p} 13.1$ were significantly down-regulated (a fold change of 3.0-3.3; $\mathrm{P}<0.05$ ) in triple-negative $\mathrm{BC}$, compared to those reported for adjacent normal tissues (29). Liu et al. demonstrated that miR-497 could act as an oncogene in several cancers and serve as a diagnostic and prognostic biomarker (20). In another study conducted by Shen et al., it was shown that there was a relative reduction in miR-497 expression in BC specimens. Furthermore, there was a negative correlation between miR-497 expression with pathological factors, TNM stage, lymphatic metastasis, tumor size, and HER2 (30).

In a study carried out by Luo et al., it was reported that the over-expression of miR-497-5p significantly prevented the proliferation, migration, and invasion and caused a G1 arrest. Furthermore, Luo et al. demonstrated that the expression of miR-497-5p in $\mathrm{BC}$ specimens was lower than that of the adjacent normal tissues $(\mathrm{P}<0.05)(31)$. Du et al. identified a reduction in miR-497-5p expression in both bladder cancer plasma and tissue specimens that could be applied as a suppressor gene in bladder cancer etiology and demonstrated that circulating miR-497$5 \mathrm{p}$ in plasma is a potential new biomarker for bladder cancer and assessed miR-497-5p level can facilitate bladder cancer cell apoptosis, but not cell cycle (32).

Moreover, Luo et al. have observed miR-497-5p as a potential prognostic marker and tumor suppressor in human cervical cancer tissues acting by posttranscriptionally targeting the insulin-like growth factor 1 (33).

According to the findings of the present study, miR-320a and miR-497-5p were down-regulated in the serum of the $\mathrm{BC}$ patients in comparison to those reported for the healthy individuals. Several studies demonstrated that the profiles of miRNAs were differentially expressed between healthy tissue and cancerous tumor samples; however, the status of the miRNAs in the serum was not reported. In the present study, two miRNAs were down-regulated in the $\mathrm{BC}$ patients in comparison to those reported for the healthy controls; nevertheless, there was no significant difference in the expression levels of these miRNAs in the early and advanced stages of BC.

There were significant differences in the expression levels of serum miRNAs in premenopausal status between the patient group and control group for miR-320a and miR-497-5p. The expression level of serum miR-320a was significantly down-regulated in patients of $<48$ years in comparison to that of the controls, but not for the patients of $\geq 48$ years. The aforementioned findings suggested that miR-320a can be regarded as a biomarker for the detection of $\mathrm{BC}$ in premenopausal subjects and women of < 48years. 
There was a relative decrease in miR-320a and miR-497-5p expression in the BC specimens, and a negative correlation was observed between miR-320a and miR-497-5p expression with clinicopathological features. The current study investigated the relationship between the levels of miR-320a and miR-497-5p with hormone receptors, PR, ER, and HER2; accordingly, there was no correlation between ER, PR, and HER2 status with miR-320a. Moreover, the aforementioned findings revealed that miR-320a probably acts independently of hormone receptors. The expression level of serum miR-497-5p was significantly down-regulated in ER status patients but not for PR and HER2 as shown in Table 3. The obtained findings demonstrated that miR-497-5p can be regarded as a biomarker for the detection of BC in premenopausal and ER patients.

Several studies reported that miR-320a and miR497-5p had different expression and could be potential diagnostic markers for cancers. To the best of our knowledge, this study has been the first attempt to fully examine the application of these serum miRNAs as a non-invasive measure for the diagnosis of early-stage BC. In the present study, miR$320 \mathrm{a}$ and miR-497-5p were reported with abnormal expression in the serum of the BC subjects. These results supported that serum miR-320a and miR497-5p could be promising new circulating biomarkers for the diagnosis of early-stage BC. It is required to carry out further studies to investigate the clinical use of serum miRNAs as promising diagnostic and prognostic biomarkers for BC.

\section{Conclusion}

According to the obtained results of the current study, it was shown that miR-320a and miR-497-5p were down-regulated in the $\mathrm{BC}$ patients in comparison to those reported for the healthy individuals. There was down-regulation in the level of miR-320a in premenopausal women and BC patients of < 48years. The expression level of miR-497-5p for ER-positive and -negative was a significant difference.

The aforementioned findings suggest the potentiality of miR-320a for the detection of $\mathrm{BC}$, particularly in premenopausal and < 48years females, and miR-497-5p could be considered a biomarker for ER-positive and -negative monitoring in early-stage $\mathrm{BC}$ patients. The present study highlights the potential role of serum circulating miR-320a and miR-497-5p expression levels for the detection of early-stage BC. It is necessary to perform further large-scale studies to confirm the results of the current study.

\section{Acknowledgments}

The authors would like to thank all patients and healthy individuals for their participation in this study.

\section{Footnotes}

Authors' contributions: Zahra Nashtahosseini and Farzin Sadeghi performed the research; Zahra Nashtahosseini and Mahmoud R. Aghamaali designed the research study; Mahmoud R. Aghamaali contributed essential reagents or tools; Mahmoud R. Aghamaali and Zahra Nashtahosseini analyzed the data; Zahra Nashtahosseini wrote the paper and all authors have read and approved the manuscript.

Conflicts of Interest: The authors declare that there is no conflict of interest.

Ethical approval: All the procedures performed in this study involving human participants were in accordance with the ethical standards of the Ethics Committee of Babol University of Medical Sciences and 1964 Declaration of Helsinki and its later amendments or comparable ethical standards (Approval ID: IR.MUBABOL.HRI.REC.1398.144) in 2019.

Informed consent: Informed consent was obtained from all the participants included in the study.

Funding/Support: The authors did not receive any funding.

\section{References}

1. Waks AG, Winer EP. Breast cancer treatment. JAMA. 2019;321(3):316. doi: 10.1001/jama.2018.20751. [PubMed: 30667503].

2. Wang L. Early diagnosis of breast cancer. Sensors (Basel). 2017;17(7):1572. doi: 10.3390/s17071572. [PubMed: 28678153].

3. Peng Y, Croce CM. The role of MicroRNAs in human cancer. Signal Transduct Target Ther. 2016;1:15004. doi: 10.1038/sigtrans.2015.4. [PubMed: 29263891].

4. O'Brien J, Hayder H, Zayed Y, Peng C. Overview of microRNA biogenesis, mechanisms of actions, and circulation. Front Endocrinol (Lausanne). 2018;9:402. doi: 10.3389/fendo. 2018.00402. [PubMed: 30123182].

5. Catalanotto C, Cogoni C, Zardo G. MicroRNA in control of gene expression: an overview of nuclear functions. Int J Mol Sci. 2016;17(10):1712. doi: 10.3390/ijms17101712. [PubMed: 27754357]

6. Iorio MV, Croce CM. MicroRNA dysregulation in cancer: diagnostics, monitoring and therapeutics. A comprehensive review. EMBO Mol Med. 2012;4(3):143-59. doi: 10.1002/ emmm.201100209. [PubMed: 22351564].

7. Jafarian AH, Kooshkiforooshani M, Farzad F, Mohamadian Roshan N. The Relationship between fibroblastic growth factor receptor-1 (FGFR1) gene amplification in triple negative breast carcinomas and clinicopathological prognostic factors. Iran J Pathol. 2019;14(4):299-304. doi: 10.30699/ijp. 2019.96713.1952. [PubMed: 31754359].

8. Wu X, Ding M, Lin J. Three-microRNA expression signature predicts survival in triple-negative breast cancer. Oncol Lett. 2020;19(1):301-8. doi: 10.3892/ol.2019.11118. [PubMed: 31897142]

9. Garzon R, Marcucci G. Potential of microRNAs for cancer diagnostics, prognostication, and therapy. Curr Opin Oncol. 2012;24(6):655-9. doi: 10.1097/CCO.0b013e328358522c. [PubMed: 23079782].

10. Tie J, Fan D. Big roles of microRNAs in tumorigenesis and tumor development. Histol Histopathol. 2011;26(10):1353-61. 
doi: 10.14670/HH-26.1353. [PubMed: 21870338].

11. Ali Syeda Z, Langden SS, Munkhzul C, Lee M, Song SJ. Regulatory mechanism of microRNA expression in cancer. Int J Mol Sci. 2020;21(5):1723. doi: 10.3390/ijms21051723 [PubMed: 32138313].

12. Baldassarre A, Felli C, Prantera G, Masotti A. Circulating microRNAs and bioinformatics tools to discover novel diagnostic biomarkers of pediatric diseases. Genes (Basel). 2017;8(9):234. doi: 10.3390/genes8090234. [PubMed: 28925938].

13. Wang B, Yang Z, Wang H, Cao Z, Zhao Y, Gong C, et al. MicroRNA-320a inhibits proliferation and invasion of breast cancer cells by targeting RAB11A. Am J Cancer Res. 2015;5(9):2719-29. [PubMed: 26609479].

14. Shen L, Li J, Xu L, Ma J, Li H, Xiao X, et al. MiR-497 induces apoptosis of breast cancer cells by targeting Bcl-w. Exp Ther Med. 2012;3(3):475-80. doi: 10.3892/etm.2011.428. [PubMed: 22969914].

15. Yang H, Yu J, Wang L, Ding D, Zhang L, Chu C, et al. MiR-320a is an independent prognostic biomarker for invasive breast cancer. Oncol Lett. 2014;8(3):1043-50. doi: 10.3892/ ol.2014.2298. [PubMed: 25120655].

16. Lieb V, Weigelt K, Scheinost L, Fischer K, Greither T, Marcou M, et al. Serum levels of miR-320 family members are associated with clinical parameters and diagnosis in prostate cancer patients. Oncotarget. 2017;9(12):10402-16. doi: 10.18632/ oncotarget.23781. [PubMed: 29535815].

17. Luo L, Yang R, Zhao S, Chen Y, Hong S, Wang K, et al. Decreased miR-320 expression is associated with breast cancer progression, cell migration, and invasiveness via targeting Aquaporin 1. Acta Biochimica Biophys Sin (Shanghai). 2018;50(5):473-80. doi: 10.1093/abbs/gmy023. [PubMed: 29538612].

18. Bai JW, Wang X, Zhang YF, Yao GD, Liu H. MicroRNA-320 inhibits cell proliferation and invasion in breast cancer cells by targeting SOX4. Oncol Lett. 2017;14(6):7145-52. doi: 10.3892/ol.2017.7087. [PubMed: 29344145].

19. Zhang T, Zou P, Wang T, Xiang J, Cheng J, Chen D, et al. Downregulation of miR-320 associated with cancer progression and cell apoptosis via targeting Mcl-1 in cervical cancer. Tumour Biol. 2016;37(7):8931-40. doi: 10.1007/s13277-015-4771-6. [PubMed: 26753959].

20. Liu Z, Wu S, Wang S, Kang S, Zhao B, He F, et al. Prognostic value of microRNA-497 in various cancers: a systematic review and meta-analysis. Dis Markers. 2019;2019:2491291. doi: 10.1155/2019/2491291. [PubMed: 31191744].

21. Hou H, Gong L, Zhou L, Qin H, Mei X, Xie Y, et al. The potential role of microRNA-497 in different cancers. Int J Clin Exp Pathol. 2016;9(8):7813-8.

22. Wu Z, Cai X, Huang C, Xu J, Liu A. MiR-497 suppresses angiogenesis in breast carcinoma by targeting HIF- $1 \alpha$.
Oncol Rep. 2016;35(3):1696-702. doi: 10.3892/or.2015.4529. [PubMed: 26718330].

23. Heydari N, Nikbakhsh N, Sadeghi F, Farnoush N, Khafri S, Bastami M, et al. Overexpression of serum microRNA-140-3p in premenopausal women with newly diagnosed breast cancer. Gene. 2018;655:25-9. doi: 10.1016/j.gene.2018.02.032. [PubMed: 29474861].

24. Nashtahosseini Z, Aghamaali MR, Sadeghi F, Heydari N, Parsian H. Circulating status of microRNAs $660-5 p$ and $210-3 p$ in breast cancer patients. J Gene Med. 2021;23(4):e3320. doi: 10.1002/jgm.3320. [PubMed: 33533518].

25. Pfaffl MW. A new mathematical model for relative quantification in real-time RT-PCR. Nucleic Acids Res. 2001; 29(9):e45. doi: 10.1093/nar/29.9.e45. [PubMed: 11328886].

26. Wang H, Peng R, Wang J, Qin Z, Xue L. Circulating microRNAs as potential cancer biomarkers: the advantage and disadvantage. Clin Epigenetics. 2018;10:59. doi: 10.1186/ s13148-018-0492-1. [PubMed: 29713393].

27. Lindner K, Haier J, Wang Z, Watson DI, Hussey DJ, Hummel R, et al. Circulating microRNAs: emerging biomarkers for diagnosis and prognosis in patients with gastrointestinal cancers. Clin Sci (Lond). 2015;128(1):1-15. doi: 10.1042/ CS20140089. [PubMed: 25168167].

28. Yan LX, Huang XF, Shao Q, Huang MY, Deng L. MicroRNA miR21 overexpression in human breast cancer is associated with advanced clinical stage, lymph node metastasis and patient poor prognosis. RNA. 2008;14(11):23-60. doi: 10.1261/ rna.1034808. [PubMed: 18812439].

29. Chang YY, Kuo WH, Hung JH, Lee CY, Lee YH, Chang YC, et al. Deregulated microRNAs in triple-negative breast cancer revealed by deep sequencing. Mol Cancer. 2015;14:36. doi: 10.1186/s12943-015-0301-9. [PubMed: 25888956].

30. Shen L, Li J, Xu L, MA J, LI H, Xiao X, et al. miR-497 induces apoptosis of breast cancer cells by targeting Bcl-w. Exp Ther Med. 2012;3(3):475-80. doi: 10.3892/etm.2011.428. [PubMed: 22969914].

31. Luo Q, Li X, Gao Y, Long Y, Chen L, Huang Y, et al. MiRNA-497 Regulates cell growth and invasion by targeting cyclin E1 in breast cancer. Cancer Cell Int. 2013;13:95. doi: 10.1186/14752867-13-95. [PubMed: 24112607].

32. Du M, Shi D, Yuan L, Li P, Chu H. Circulating miR-497 and miR$663 \mathrm{~b}$ in plasma are potential novel biomarkers for bladder cancer. Sci Rep. 2013;5:10437. doi: 10.1038/srep10437. [PubMed: 26014226].

33. Luo M, Shen, D, Zhou, $X$, Chen, $X$, Wang W. MicroRNA-497 is a potential prognostic marker in human cervical cancer and functions as a tumor suppressor by targeting the insulin-like growth factor 1 receptor. Surgery. 2013;153(6):836-47. doi: 10.1016/j.surg.2012.12.004. [PubMed: 23453369]. 